\title{
RECONSTRUCTION OF ULTRASONIC SOUND VELOCITY AND ATTENUATION COEFFICIENT USING LINEAR ARRAYS: CLINICAL ASSESSMENT
}

\author{
Chen-Han Chang, * Sheng-Wen Huang, ${ }^{*}$ Hsin-Chia Yang, ${ }^{*}$ Yi-Hong Chou, ${ }^{\dagger}$ \\ AND PAI-CHI LI** \\ *Department of Electrical Engineering, National Taiwan University; †Department of Radiology, Taipei Veterans \\ General Hospital and National Yang Ming University School of Medicine; and ${ }^{\ddagger}$ Graduate Institute of Biomedical \\ Electronics and Bioinformatics, National Taiwan University, Taipei, Taiwan
}

(Received 2 November 2006, revised 11 May 2007, in final form 16 May 2007)

\begin{abstract}
The aim of this study was to determine the efficacy of using sound velocity and tissue attenuation to clinically discriminate breast cancer from healthy tissues. The methods for reconstructing the sound-velocity and attenuation-coefficient distributions were previously proposed and tested on tissue-mimicking phantoms. The methods require only raw channel data acquired by a linear transducer array and can therefore be implemented on existing clinical systems. In this paper, these methods are tested on clinical data. A total of 19 biopsy-proven cases, consisting of five carcinomas (CAs), seven fibroadenomas (FAs), six adipose tissue (fat) and one oil cyst, were evaluated. A single imaging setup consisting of a $5-\mathrm{MHz}, 128$-channel linear array was used to simultaneously obtain B-mode image data, time-of-flight data and attenuation data. The sound velocity and attenuation coefficient can be reconstructed inside and outside a region of interest manually selected in the B-mode image. To reduce distortion caused by tissue inhomogeneities, an optimal filter derived from pulse-echo data-with water replacing the breast tissue-is applied. We found that the sound velocities in CA, FA and fat tissues relative to those in the surrounding tissues were $49.8 \pm 35.2,2.6 \pm 27.3$ and $-25.1 \pm 44.9 \mathrm{~m} / \mathrm{s}(\mathrm{mean} \pm \mathrm{SD})$, respectively, whereas the relative attenuation coefficients were $0.21 \pm 0.58,0.27 \pm 0.62$ and $-0.02 \pm 0.59 \mathrm{~dB} / \mathrm{cm} / \mathrm{MHz}$. These results indicate that $\mathrm{CA}$ can be discriminated from $\mathrm{FA}$ and fat by choosing an appropriate threshold for the relative sound velocity (i.e., $18.5 \mathrm{~m} / \mathrm{s}$ ). However, the large variations in the attenuation within the same type of tissue make simple thresholding ineffective. Nevertheless, the method described in this paper has the potential to reduce negative biopsies and to improve the accuracy of breast cancer detection in clinics. (E-mail: paichi@cc.ee.ntu.edu.tw) (c) 2007 World Federation for Ultrasound in Medicine \& Biology.
\end{abstract}

Key Words: Time-of-flight, Attenuation, Region-of-interest, Sound velocity, Attenuation coefficient.

\section{INTRODUCTION}

Breast cancer is a leading cause of death from cancer in females, indicating the importance of its early detection. Mammography is considered the best noninvasive screening method because it can detect nonpalpable and small tumors. Nevertheless, mammography exposes the subject to ionizing radiation and the mammograms of young women with dense breasts are hard to interpret (Lanfranchi 2000). Ultrasonic imaging has become an effective adjunct to mammography (Telger 1996), but the use of conventional ultrasonic pulse-echo B-mode imaging to find breast tumors is also often limited by the

Address correspondence to: Pai-Chi Li, Department of Electrical Engineering, National Taiwan University, No. 1, Sec. 4, Roosevelt Road, Taipei 106, Taiwan. E-mail: paichi@cc.ee.ntu.edu.tw image distortion caused by sound-velocity inhomogeneities in the breast tissue (Gauss et al. 1997). With ultrasound, it is also possible to detect breast cancer by reconstructing distributions of the sound velocity and attenuation coefficient. For example, it has been reported that the sound velocity is faster in cancerous tissue than in fat (Goss et al. 1978) and that the attenuation coefficient is higher in cancerous tissue than in a cyst (Goss et al. 1980; Madsen et al. 1982, 1988). However, these reconstructions typically require a tomographic setup to acquire data from a large number of projections, and such setups are rarely used in clinical situations. Another approach to the tomographic reconstructions of sound velocity and attenuation coefficient is limited-angle tomography using linear arrays (Huang and Li 2004a, 2004b, 2005; Krueger et al. 1998). This uses the same 
imaging setup as that used for conventional B-mode imaging and, thus, both imaging methods can be used. In addition, a plate reflector is placed under the breast, and the breast is under compression during data acquisition. This approach also requires the selection of a region-ofinterest (ROI) to alleviate the difficulties caused by the limited angles of tomographic data acquisition, and it has yielded good results on tissue-mimicking phantoms (Huang and Li 2004b, 2005). In the present study we applied the approach to clinical data, and we also modified the original method to accommodate tissue inhomogeneities that are present in breast tissue (which were not present in the phantoms used previously). Because a critical step in this approach is preselecting an ROI, the effects of variations in the ROI selection on the performance of the method were also determined.

\section{METHODS}

This section first introduces the method for reconstructing the distributions of sound velocity and attenuation coefficient, and then describes the experimental setup for acquiring clinical data from individual array channels. We then list the procedures of ROI selection and the implementation of a least-squares (LS) filter used to reduce distortion from tissue inhomogeneities and then, finally, we describe the patients.

\section{Reconstruction of sound velocity and attenuation coefficient}

Based on our previous studies (Huang and Li 2004a, 2004b, 2005) and Kossoff et al. (1973), water was used as a reference, whereby for each clinical dataset, a corresponding dataset acquired with water replacing breast tissue was also acquired without changing the setup. The sound velocity of water $\left(c_{0}\right)$ was assumed to be $1496 \mathrm{~m} / \mathrm{s}$ (Bilaniuk and Wong 1993) and the attenuation coefficient of water $\left(\alpha_{0}\right)$ was specified as $0 \mathrm{~dB} / \mathrm{cm} / \mathrm{MHz}$ at room temperature, $25^{\circ} \mathrm{C}$. As described below, the known properties of water were used to estimate the properties of the breast. Let $c_{1}(x, y)$ denote the sound velocity at the position $(x, y)$ in the B-mode image. As shown in Fig. 1, if the ultrasonic signal has round-trip times-of-flight $t_{0}$ and $t_{1}$ in water and breast, respectively, then

$$
\begin{gathered}
t_{0}=\int_{L} \frac{1}{c_{0}} d l=\int_{L} s_{0} d l, \\
t_{1}=\int_{L} \frac{1}{c_{1}(x, y)} d l=\int_{L} s_{1}(x, y) d l,
\end{gathered}
$$

where $L$ is the signal path length and $s_{1}(x, y)$ is defined as the slowness. The difference between $t_{0}$ and $t_{1}$ is

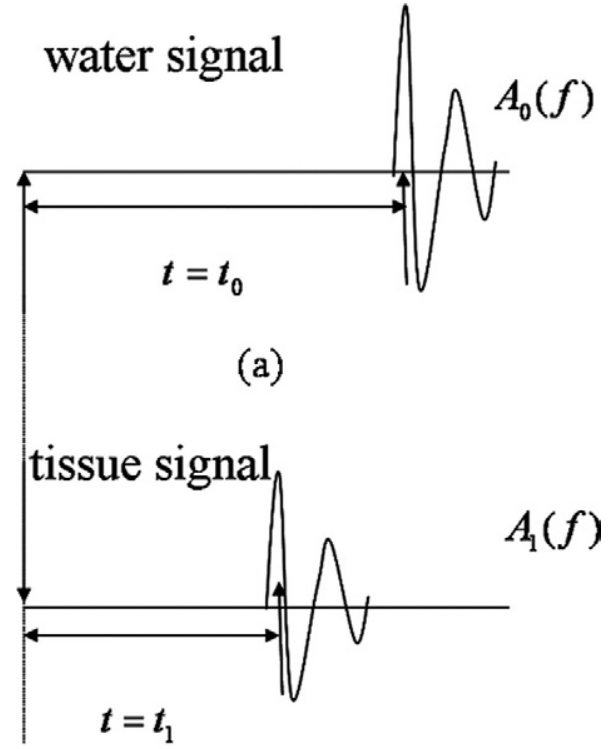

(b)

Fig. 1. Illustration of the time-of-flight and tissue attenuation of the water signal (top) and the breast signal (bottom).

$$
\Delta t=t_{1}-t_{0}=\int_{L}\left[s_{1}(x, y)-s_{0}\right] d l=\int_{L} \Delta s(x, y) d l .
$$

The attenuation coefficient is linearly proportional to frequency in the frequency ROI and represented by $\alpha(x, y)$. Let $A_{0}(f)$ and $A_{1}(f)$ denote the echo spectra for water and breast as the propagating media, respectively, then

$$
\begin{array}{r}
\Delta A(f)=20 \log _{10}\left(A_{0}(f)\right)-20 \log _{10}\left(A_{1}(f)\right) \\
\Delta A^{\prime}=\int_{L} \alpha(x, y) d l \quad(\mathrm{~dB} / \mathrm{MHz}),
\end{array}
$$

where $\Delta A^{\prime}$ is also the slope of a first-order polynomial fit to $\Delta A(f)$.

The integral equations previously mentioned (i.e., eqns (3) and (5)) can be written in the following discrete forms:

$$
\begin{gathered}
\Delta t_{i j}=\sum_{n=1}^{N} \sum_{m=1}^{M} \Delta s(m, n) l_{i j}(m, n), \\
\Delta A^{\prime}{ }_{i j}=\sum_{n=1}^{N} \sum_{m=1}^{M} \alpha(m, n) l_{i j}(m, n),
\end{gathered}
$$

where the B-mode image is divided into $N \times M$ pixels, and the index $i j$ represents the transmit-receive channel 


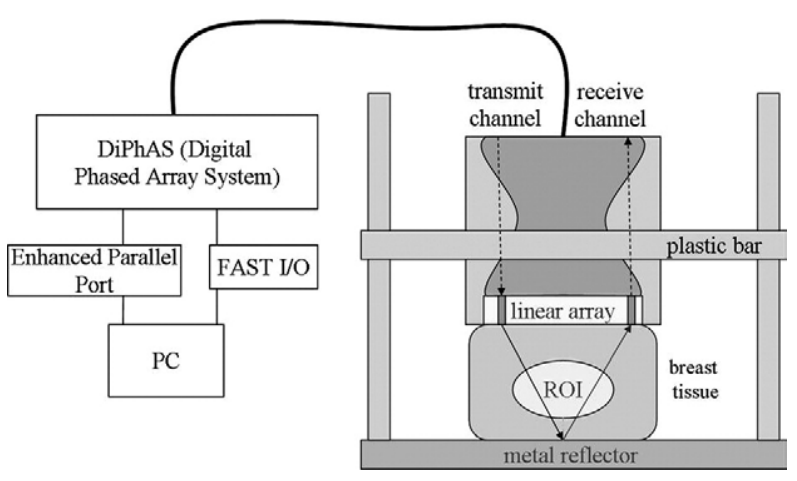

Fig. 2. Schematic of the setup for acquiring clinical data. The plastic bar was used to hold the transducer. The setup was used

to compress the breast, similar to X-ray mammography.

combination (i.e., transmit from the $i^{\text {th }}$ channel and receive on the $j^{\text {th }}$ channel). Furthermore, eqns (6) and (7) can be written in matrix form as

$$
\begin{aligned}
& L \Delta s=\Delta t, \\
& L \alpha=\Delta A^{\prime} .
\end{aligned}
$$

Note that the dimensions of the matrices and vectors in eqns (8) and (9) can be reduced by discarding data containing apparent errors. For example, if the time-offlight difference is outside a prespecified reasonable range, it is discarded. The reasonable range for the timeof-flight is determined by assuming the sound velocity to be within the range 1400 to $1600 \mathrm{~m} / \mathrm{s}$, because the sound velocities of cyst and fat are about $1570 \mathrm{~m} / \mathrm{s}$ and 1470 $\mathrm{m} / \mathrm{s}$, respectively. The details of this approach are available elsewhere (Huang and Li 2004a, 2004b, 2005).

\section{Data acquisition}

Figure 2 shows a schematic of the experimental setup used to acquire clinical data. The plastic bar was used to hold the transducer. The setup was used to compress the breast, similar to X-ray mammography. A programmable digital phased-array system capable of acquiring radiofrequency channel data (DiPhAS, Fraunhofer IBMT, St. Ingbert, Germany) was used (Lemor et al. 2003). The full dataset includes radiofrequency pulse-echo data from all individual channels with all transmit-receive combinations. The linear array transducer (L6/128, STI, State College, PA, USA) had 128 channels with a pitch of $0.3 \mathrm{~mm}$, an elevation width of 5 $\mathrm{mm}$, an elevation focus of $25 \mathrm{~mm}$, a center frequency of $5.57 \mathrm{MHz}$ and a $-6-\mathrm{dB}$ bandwidth of $4.10 \mathrm{MHz}$. The transmitted pulse is a five-cycle square wave at $5 \mathrm{MHz}$, and all data were sampled at $20 \mathrm{MHz}$, with a vertical resolution of 12 bits. The collected channel data were acquired at a sampling rate of $20 \mathrm{MHz}$ using a digital I/O card (PCI-7300A, ADLINK, Taipei, Taiwan) housed in a personal computer. Furthermore, a stainless plate was placed at the bottom as a reflector so that limited-angle tomographic data could be acquired. The total data acquisition time was around $4 \mathrm{~s}$, during which the patients were asked to hold their breath to reduce motion artifacts.

After acquiring a full dataset, the first step was to extract from the data to estimate the difference in timeof-flights $t_{0}$ and $t_{1}$ based on eqns (1) through (3). The second step was to estimate attenuation based on eqns (4) and (5). As mentioned in "Reconstruction of Sound Velocity and Attenuation Coefficient" section, following eqns (6) and (7), the final matrix forms of time-of-flight data and attenuation data were produced.

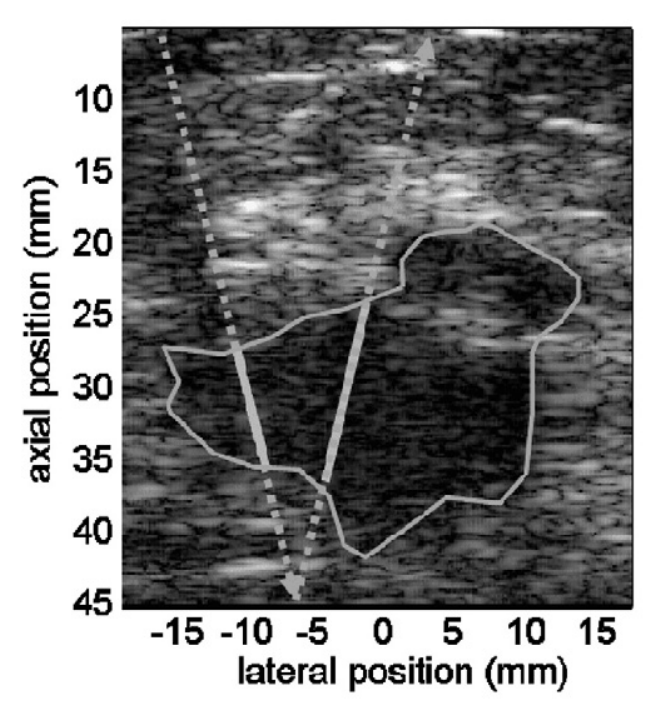

(a)

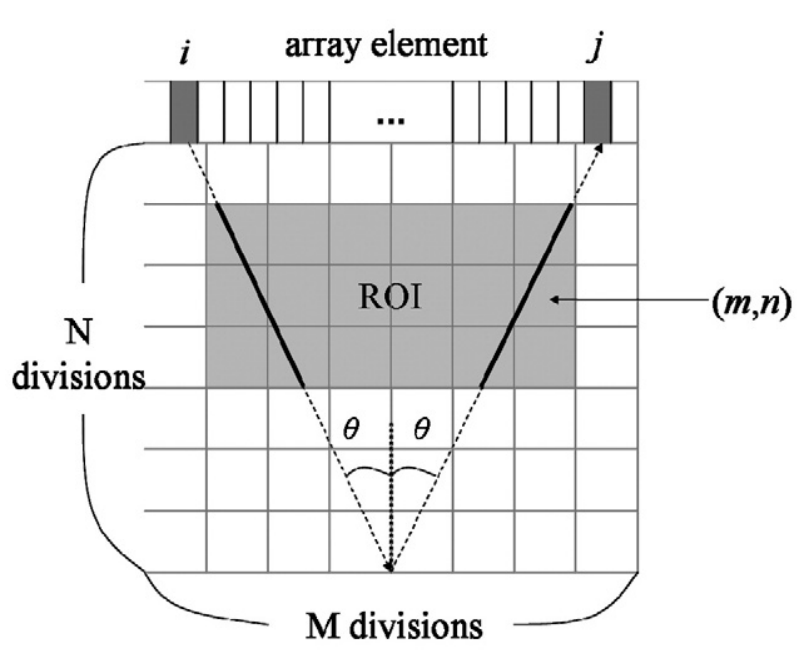

(b)

Fig. 3. An example of (a) ROI selection and (b) the schematic of signal path of one transmit-receive combination. 


\section{Region of interest selection}

For each dataset, an ROI such as the one shown in Fig. 3a was preselected manually using a graphical user interface. According to Chiang et al. (2001), some contour features, such as spiculation and angular margin, have been recognized as characteristics of malignancy. The clinician chose the ROIs according to the primary features associated malignancy such as echogenicity, size, shape and the border of the tumors. Some additional contour features, such as spiculation and angular margin, have also been used. The purpose of ROI selection is to provide reconstruction constraints by forcing both the sound velocity and attenuation coefficient values to be uniform inside and outside the ROI (Huang and Li 2005). The assumption of uniformity can be seen as the average for that region. A technique for measuring the in-vivo average velocity of ultrasound in the female breasts was developed in Kossoff et al. (1973). In Fig. 3a and b, the thick solid line represents the ROI path, whereas the dashed line is for the background path. In Fig. 3b, it shows that the signal is transmitted from the $i^{\text {th }}$ channel and received by the $j^{\text {th }}$ channel. Based on the aforementioned constraints, eqns (6) and (7) can be reduced to

$$
\begin{array}{r}
\Delta t_{i j}=\Delta S_{R O I} \sum_{n=1}^{N} \sum_{m=1}^{M} l_{i j, R O I}(m, n)+\Delta s_{\text {back }} \sum_{n=1}^{N} \sum_{m=1}^{M} l_{i j, b a c k}(m, n) \\
=l_{i j, R O I} \Delta s_{R O I}+l_{i j, b a c k} \Delta s_{\text {back }},
\end{array}
$$

$$
\begin{array}{r}
\Delta A^{\prime}{ }_{i j}=\alpha_{R O I} \sum_{n=1}^{N} \sum_{m=1}^{M} l_{i j, R O I}( \\
=l_{i j, R O I} \alpha_{R O I}+l_{i j, b a c k} \alpha_{b a c k} .
\end{array}
$$

where $(m, n)$ corresponds to one pixel in the B-mode image. When $i$ is not equal to $j, \theta$ is not equal to zero degree. Also, eqns (8) and (9) are modified to

$$
\begin{aligned}
& {\left[\begin{array}{ll}
l_{R O I} & l_{\text {back }}
\end{array}\right]\left[\begin{array}{c}
\Delta s_{R O I} \\
\Delta s_{b a c k}
\end{array}\right]=\Delta t,} \\
& {\left[\begin{array}{ll}
l_{R O I} & l_{b a c k}
\end{array}\right]\left[\begin{array}{c}
\alpha_{R O I} \\
\alpha_{b a c k}
\end{array}\right]=\Delta A^{\prime},}
\end{aligned}
$$

where $l_{R O I}, l_{\text {back }}, \Delta t$ and $\Delta A^{\prime}$ are column vectors, with each element therein representing a valid transmissionreception combination. $l_{R O I}$ is the total length of the ROI path and $l_{\text {back }}$ is the total length of the background path, $\Delta s$ is the difference in slowness, $\alpha$ is the attenuation coefficient, $\Delta t$ is the estimated difference in the timesof-flight and $\Delta A^{\prime}$ is the estimated attenuation at the center frequency of $5 \mathrm{MHz}$.

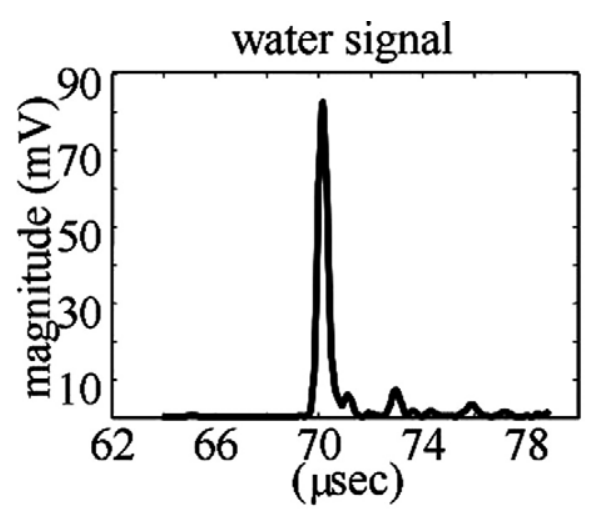

(a)

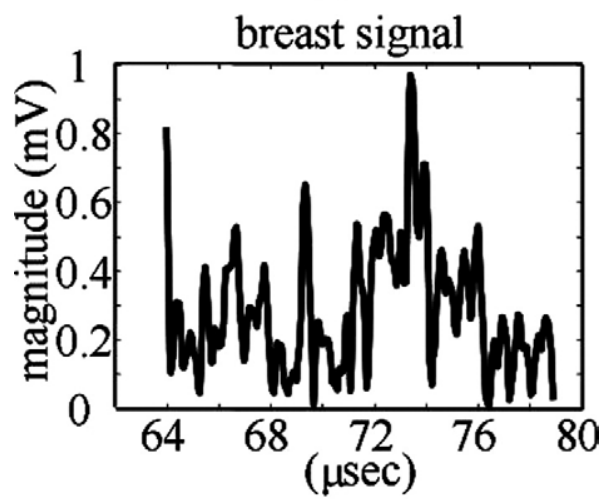

(b)

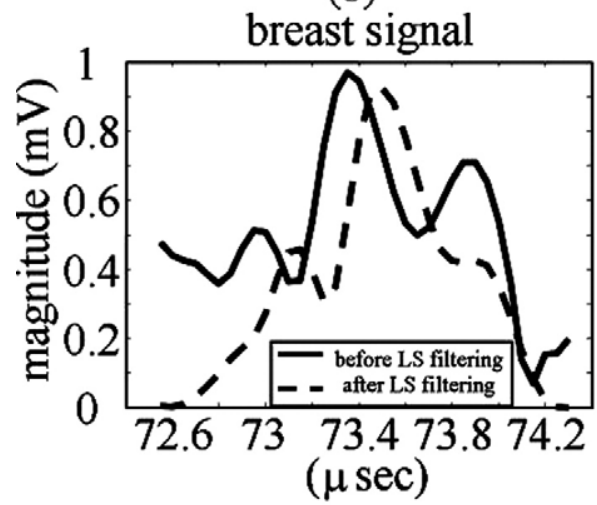

(c)

Fig. 4. Envelopes of the received signals, which are the first echo reflected from metal reflector: (a) water, (b) breast and (c) breast before (thick solid line) and after LS filtering (dashed line). The distortion from the breast tissue is clearly shown as the peak position is shifted, waveform is changed and the clutter signal is higher.

\section{Least-squares filter for distortion reduction}

Due to the inhomogeneities present in breast tissue, the received signal is often severely distorted in both amplitude and phase compared with those of a water signal (Lewin and O'Brien 1996; Zhu and Steinberg 1992, 1994, 1996, 1997). A typical example is shown in Fig. 4a (envelope of water signal with no distortion) and 
Fig. $4 \mathrm{~b}$ (envelope of breast signal with distortion). Figure $4 \mathrm{a}$ and $\mathrm{b}$ show the first echo reflected from the metal plate through water and breast tissue, respectively. Compared with the water signal (Fig. 4a), the distortion from the breast tissue is clearly shown as the peak position is shifted, waveform is changed and the clutter signal is higher. Such distortion significantly affects the estimation results, and an LS filter was used to reduce potential errors. For each transmit-receive combination, the filter was designed by using the breast signal as the input and the corresponding water signal as the desired output. In addition, the DC gain of the filter was normalized to unity to not introduce errors into the attenuation estimation. Likewise, the linear component of the phase in the frequency domain was also removed to not introduce additional time delays that would affect the time-offlight.

Letting $b$ denote the breast signal vector, whose envelope is as shown in Fig. 4c (the thick solid line), $w$ denotes the water signal, $f$ denotes the filter, we have

$$
b \otimes f=w,
$$

where $\otimes$ is the convolution operator. Equation (14) is further simplified into a multiplication form as

$$
B \cdot f=w,
$$

where $B$ is the convolution matrix of $b$ (Li et al. 1992). According to the LS error method, $f$ can be obtained from

$$
f=\left(B^{\prime} B\right)^{-1} B^{\prime} w .
$$

Note that the above equations are based on the baseband signal representation. As mentioned previously, filter $f$ was modified to not introduce estimation errors: it was first normalized to its DC gain and then the first-order (i.e., linear) component of the phase in the frequency domain was removed.

Figure $4 \mathrm{c}$ shows the filtered version (the dashed

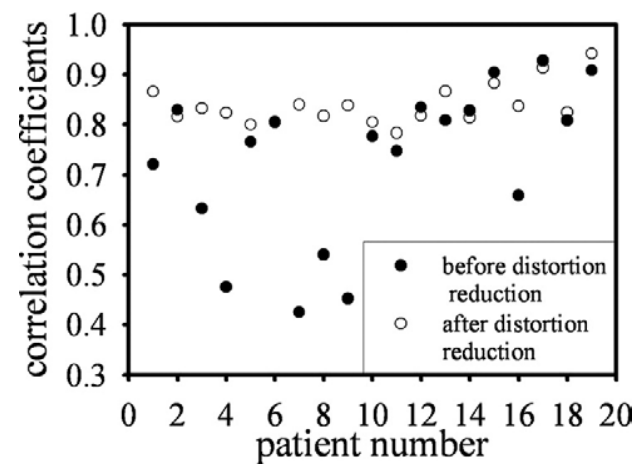

Fig. 5. Correlation coefficients before and after distortion reduction. The correlation coefficients of case 6 were virtually indistinguishable.

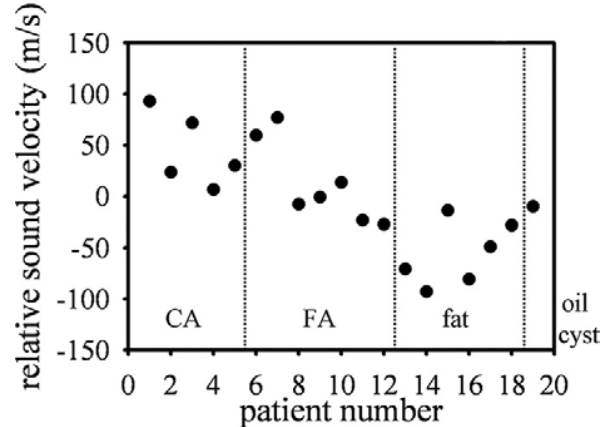

Fig. 6. Relative sound-velocity results.

line) of the breast signal (the thick solid line), which indicates that the filtering allows the time-of-flight and attenuation coefficient to be estimated more accurately. From Waag et al. (1989), the received signal can be represented by a convolution of an undistorted beam and a distortion function. Therefore, the correlation coefficient can represent the extent of distortion. The efficacy of the LS filter is further illustrated by the correlation coefficients between the breast signal (with and without filtering) and the water signal as shown in Fig. 5. It shows that these correlation coefficients are generally higher than 0.7 after filtering, whereas before filtering some of the correlation coefficients are low and the estimation results are not reliable.

\section{Patients}

Nineteen biopsy-proven cases, consisting of five carcinomas (CAs), seven fibroadenomas (FAs), six adipose tissue (fat) and one oil cyst, were evaluated. The clinical data were collected in the ultrasonic consulting room at Taipei Veterans General Hospital by one of the authors after patients had provided informed consent. During data acquisition, the patients (in a sitting position) were asked to hold their breath and their breasts were compressed by the device shown in Fig. 2.

\section{RESULTS AND DISCUSSION}

In the following, the relative values (i.e., difference between the ROI and the background) were used.

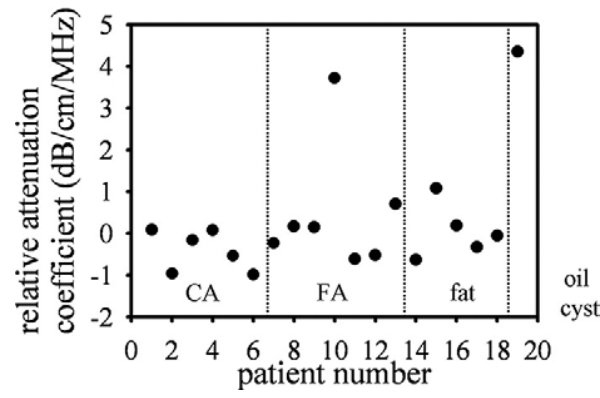

Fig. 7. Relative attenuation-coefficient results. 
Table 1. Average sound velocity and attenuation coefficient with SD

\begin{tabular}{lcc}
\hline & $\begin{array}{c}\text { Sound velocity } \\
(\mathrm{m} / \mathrm{s})\end{array}$ & $\begin{array}{c}\text { Attenuation coefficient } \\
(\mathrm{dB} / \mathrm{cm} / \mathrm{MHz})\end{array}$ \\
\hline $\mathrm{CA}$ & $1530.9 \pm 36.2$ & $0.66 \pm 0.52$ \\
FA & $1499.8 \pm 26.8$ & $0.68 \pm 0.66$ \\
Fat & $1464.8 \pm 45.1$ & $0.63 \pm 0.61$ \\
\hline
\end{tabular}

The relative sound velocities are shown in Fig. 6 and were $49.8 \pm 35.2 \mathrm{~m} / \mathrm{s}$ (mean $\pm \mathrm{SD}), 2.6 \pm 27.3 \mathrm{~m} / \mathrm{s}$ and $-25.1 \pm 44.9 \mathrm{~m} / \mathrm{s}$ for CA, FA and fat, respectively. The relative sound velocity for the single case of an oil cyst was $-9.4 \mathrm{~m} / \mathrm{s}$. According to Fig. 6 , it is possible to correctly distinguish 14 of 18 cases (excluding the oil cyst) using thresholds of $18.5 \mathrm{~m} / \mathrm{s}$ and $-28.3 \mathrm{~m} / \mathrm{s}$. The relative attenuation coefficients are shown in Fig. 7 and were $0.21 \pm 0.58,0.27 \pm 0.62$ and $-0.02 \pm 0.59$ $\mathrm{dB} / \mathrm{cm} / \mathrm{MHz}$ for CA, FA and fat, respectively. It demonstrates that, unlike sound velocity, it is not feasible to use the relative attenuation coefficient to distinguish different types of objects. On the other hand, the average results of sound velocity and attenuation coefficients were put in Table 1.

Effects of different reference sound velocity were also investigated. As shown in Table 2, using $1522 \mathrm{~m} / \mathrm{s}$ as the sound velocity in water, the relative sound velocities are $44.9 \pm 35.9 \mathrm{~m} / \mathrm{s}$ (mean $\pm \mathrm{SD}), 13.0 \pm 40.5 \mathrm{~m} / \mathrm{s}$ and $-55.9 \pm 30.9 \mathrm{~m} / \mathrm{s}$ for CA, FA and fat, respectively. The sound velocity thresholds can be set at $17.6 \mathrm{~m} / \mathrm{s}$ and $-39.3 \mathrm{~m} / \mathrm{s}$, respectively. Similarly, the relative attenuation coefficients using $1522 \mathrm{~m} / \mathrm{s}$ as the sound velocity in water, as shown in Table 3 , are $-0.30 \pm 0.45,0.24 \pm$ 1.59 and $0.16 \pm 0.64 \mathrm{~dB} / \mathrm{cm} / \mathrm{MHz}$ for $\mathrm{CA}, \mathrm{FA}$ and fat, respectively.

From the tables, we found that the relative sound velocities changed by $-4.9 \mathrm{~m} / \mathrm{s},-10.4 \mathrm{~m} / \mathrm{s}$ and +30.8 $\mathrm{m} / \mathrm{s}$ for CA, FA and fat, respectively. Nonetheless, 14 of 18 cases can be distinguished in both cases by choosing threshold individually. Also, it showed that the temperature control during data acquisition is critical.

Because the ROI was selected manually, the impact of ROI selection on the estimation results was also evaluated. Figure 8a and b show, respectively, the sound-

Table 2. Relative sound velocity at different reference sound velocities

\begin{tabular}{rcrr}
\hline $\begin{array}{c}\text { Relative sound } \\
\text { velocity }(\mathrm{m} / \mathrm{s})\end{array}$ & CA & FA & \multicolumn{1}{c}{ Fat } \\
\hline I. $\mathrm{c}_{0}=1496 \mathrm{~m} / \mathrm{s}$ & $49.8 \pm 35.2$ & $2.6 \pm 27.3$ & $-25.1 \pm 44.9$ \\
II. $\mathrm{c}_{0}=1522 \mathrm{~m} / \mathrm{s}$ & $44.9 \pm 35.9$ & $13.0 \pm 40.5$ & $-55.9 \pm 30.9$ \\
\hline
\end{tabular}

Table 3. Relative attenuation coefficient at two reference sound velocities

\begin{tabular}{lrcr}
\hline $\begin{array}{c}\text { Relative attenuation } \\
\text { coefficient } \\
(\mathrm{dB} / \mathrm{cm} / \mathrm{MHz})\end{array}$ & CA & FA & \multicolumn{1}{c}{ Fat } \\
\hline I. $\mathrm{c}_{0}=1496 \mathrm{~m} / \mathrm{s}$ & $0.21 \pm 0.58$ & $0.27 \pm 0.62$ & $-0.02 \pm 0.59$ \\
II. $\mathrm{c}_{0}=1522 \mathrm{~m} / \mathrm{s}$ & $-0.30 \pm 0.45$ & $0.24 \pm 1.59$ & $0.16 \pm 0.64$ \\
\hline
\end{tabular}

velocity and attenuation-coefficient results for patients 1 , 7 and 9 as examples. The estimation results for the background generally do not change with ROI areas in the range 10 to $140 \mathrm{~mm}^{2}$. However, the attenuation coefficient of ROI for patient 7 is an exception. This may indicate that the assumption that the attenuation coefficient is uniform inside the ROI is invalid in this case.

As shown in Table 1, the sound-velocity and attenuation-coefficient results reported in this paper differ from those reported in the literature. In Duck (1990), the sound velocities for CA and FA were $1550 \pm 32 \mathrm{~m} / \mathrm{s}$ and $1584 \pm 27 \mathrm{~m} / \mathrm{s}$, and in Landini et al. (1985), the attenuation coefficients for CA, FA and fat were in the range 0.08 to $0.16 \mathrm{~dB} / \mathrm{cm} / \mathrm{MHz}$. In Huang and $\mathrm{Li} 2005$, for each transmit-receive combination, data corresponding

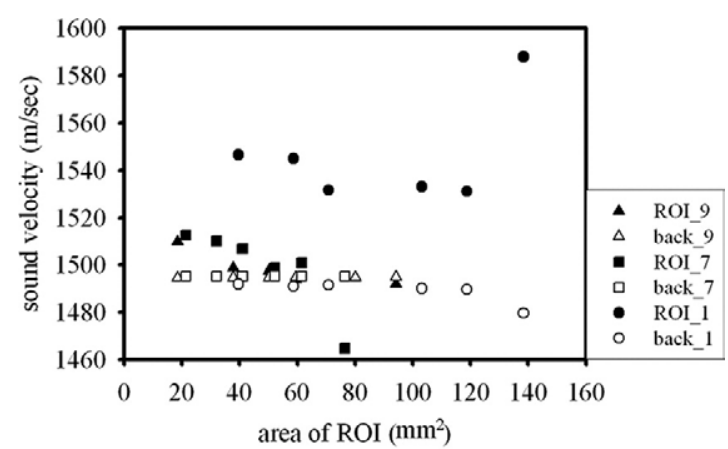

(a)

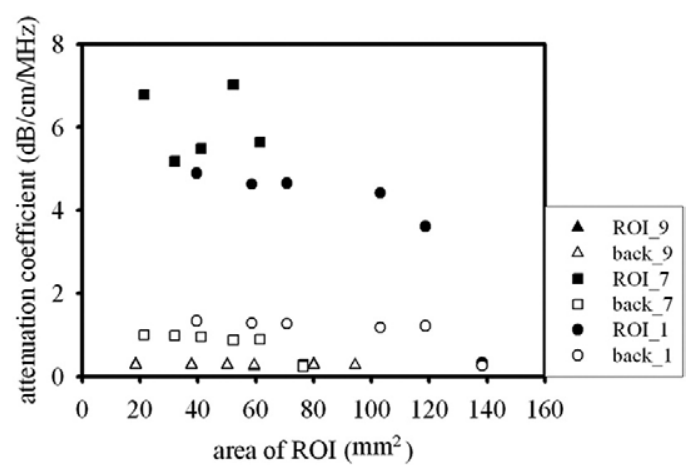

(b)

Fig. 8. (a) Estimated sound velocity and (b) attenuation coefficient as functions of the ROI area for patients 1,7 and 9 . 
to 16 consecutive firings were averaged off-line to increase the signal-to-noise ratio. However, considering the duration of patients' holding their breath, no averaging was performed in the data acquisition. Low signalto-noise ratio might lead to estimation errors. Besides, in Landini et al. (1985), each one-way measurement was obtained through 1-cm-thick tissue specimens to estimate the attenuation coefficient. Particularly, the mean value was obtained among a series of 20 measurements. In contrast, the tissue thickness under our experimental setup is usually between $7 \mathrm{~cm}$ and $10 \mathrm{~cm}$ (round trip). The standard deviations of attenuation coefficient in four groups from Landini et al. (1985) were 0.04, 0.06, 0.06 and $0.10 \mathrm{~dB} / \mathrm{cm} / \mathrm{MHz}$, respectively. On the other hand, as shown in Table 1, the standard deviations of the attenuation coefficient were from $0.52-0.66 \mathrm{~dB} / \mathrm{cm} / \mathrm{MHz}$ in our study. The poor signal-to-noise ratio because of lack of signal averaging and large propagation distance are expected to be a major factor in the large estimation errors. More patient data need to be collected and in-vivo measurements are needed to understand such differences.

The limited angle tomography use in this study is a trade-off between full-view (i.e., full $360^{\circ}$ ) tomography and direct reflection mode (i.e., only at $0^{\circ}$ ) data acquisition. Compared with full-view tomography, limited-angle tomography has loss of angular information. However, one advantage is that such a setup can be implemented easily using an existing B-mode imaging system with a linear array. To overcome the loss of information because of limited angular extent, the reconstruction method proposed in our previous studies (Huang and Li 2004a, 2004b, 2005) was adopted.

\section{CONCLUSIONS}

This study has demonstrated the feasibility of using clinical data to reconstruct the sound velocity and attenuation coefficient using a linear array system. The setup is similar to that of X-ray mammography, with the breast being compressed and LS filtering used to reduce distortion. We also evaluated the effects of changing the ROI area. Our results show that the sound velocity can be used to distinguish different lesion types, whereas the attenuation coefficient varies over a wide range and hence is not yet useful for tissue characterization. The method proposed in this study has the potential to aid breast cancer detection and decrease the percentage of negative biopsies performed in clinics.

Acknowledgments-This study was financially supported by the National Science Council of Taiwan under grant no. NSC-93-2213-E-002063.

\section{REFERENCES}

Bilaniuk N, Wong GSK. Speed of sound in pure water as a function of temperature. J Acoust Soc Am 1993;93(3):1609-1612.

Chiang HK, Tiu CM, Hung GS, Wu SC, Chang TY, Chou YH. Stepwise logistic regression analysis of tumor contour features for breast ultrasound diagnosis. IEEE Ultrason Sympos 2001;2: 1303-1306.

Duck FA. Physical properties of tissue. London: Academic Press, 1990.

Gauss RC, Soo MS, Trahey GE. Wavefront distortion measurements in the human breast. IEEE Ultrason Sympos 1997;2:1547-1551.

Goss SA, Johnston RL, Dunn F. Comprehensive compilation of empirical ultrasonic properties of mammalian tissues. J Acoust Soc Am 1978;64(2):423-457.

Goss SA, Johnston RL, Dunn F. Compilation of empirical ultrasonic properties of mammalian tissues. II. J Acoust Soc Am 1980;68(1): 93-108.

Huang SW, Li PC. Computed tomography sound velocity reconstruction using incomplete data. IEEE Trans Ultrason Ferroelectr Freq Control 2004a;51(3):329-342.

Huang SW, Li PC. Experimental investigation of computed tomography sound velocity reconstruction using incomplete data. IEEE Trans Ultrason Ferroelectr Freq Control 2004b;51(9):1072-1081.

Huang SW, Li PC. Ultrasonic computed tomography reconstruction of the attenuation coefficient using a linear array. IEEE Trans Ultrason Ferroelectr Freq Control 2005;52(11):2011-2022.

Kossoff G, Kelly-Fry E, Jellins J. Avergae Velocity of Ultrasound in the Human Female Breast. J Acoust Soc Am 1973;53(6):17301736.

Krueger M, Burow V, Hiltawsky KM, Ermert H. Limited angle ultrasonic transmission tomography of the compressed female breast. IEEE Ultrason Sympos 1998;2:1345-1348.

Landini L, Sarnelli R, Squartini F. Frequency-dependent attenuation in breast tissue characterization. Ultrasound Med Biol 1985;11(4): 599-603.

Lanfranchi ME. Breast ultrasound. 2nd ed. New York: Marban Books, 2000.

Lemor RM, Weber PK, Fonfara PK, Guenther C, Welsch JH, Hoss ML. A new combined open research platform for ultrasound radio frequency signal processing. IEEE Ultrason Sympos 2003;1:33-37.

Li PC, Ebbini E, O'Donnell M. A new filter design technique for coded excitation systems. IEEE Trans Ultrason Ferroelectr Freq Control 1992;39(6):693-699.

Lewin T, O'Brien WD Jr. Tissue-induced ultrasonic wavefront distortion. IEEE Ultrason Sympos 1996;2:1415-1418.

Madsen EL, Zagzebski JA, Frank GR, Greenleaf JF, Carson PL. Anthropomorphic breast phantoms for assessing ultrasonic imaging system performance and for training ultrasonographers: Part I. J Clin Ultrasound 1982;10(2):67-75.

Madsen EL, Kelly-Fry E, Frank GR. Anthropomorphic phantoms for assessing systems used in ultrasound imaging of the compressed breast. Ultrasound Med Biol 1988;14(suppl 1):183-201.

Telger TC. Teaching atlas of breast ultrasound. New York: Thieme Medical Publishers, 1996.

Waag RC, Smith JF 3rd, Sumino Y. Wavefront distortion in ultrasonic imaging. Proc 11th Annu Int Conf IEEE Engineering in Med and Biol Soc 1989;395-396.

Zhu Q, Steinberg BD. Large-transducer measurements of wavefront distortion in the female breasts. Ultrason Imaging 1992;14(3):276299.

Zhu Q, Steinberg BD. Modeling, measurement and correction of wavefront distortion produced by breast specimens. IEEE Ultrason Sympos 1994;3:1613-1617.

Zhu Q, Steinberg BD. A toward inverse filtering approach for ultrasonic wavefront compensation. IEEE Ultrason Sympos 1996;2: 1357-1361.

Zhu Q, Steinberg BD. Modeling and correction of incoherent wavefront distortion. Int J Imag Syst Technol 1997;8(3):322-335. 